\title{
Sedimentation of gas-fluidized particles with random shape and size
}

\author{
Laurence Girolami* \\ Laboratoire GéHCO, Campus Grandmont, \\ Université de Tours, 37200 Tours, France
}

Frédéric Risso ${ }^{\dagger}$

Institut de Mécanique des Fluides de Toulouse (IMFT), Université de Toulouse, CNRS, 31400 Toulouse, France

(Dated: March 4, 2019) 


\begin{abstract}
This work deals with the fluidization and sedimentation of fine solid particles, of random shape and size, similarly to those commonly involved in geophysical mass flows, such as pyroclastic flows. While heated to avoid the effect of moisture and the formation of clusters, particles were first uniformly fluidized by a hot gas flow, up to a high expansion rate, then let sedimented after stopping the gas supply. Three different materials are explored, involving contrasted geometries, each characterized by a specific particle volume fraction at packing $\Phi_{\text {pack }}$. Within the range of values of the solid volume fraction $\Phi_{s} / \Phi_{\text {pack }}$ studied here, from 0.65 to 0.95 , the dense suspension forms a fully fluidized homogeneous mixture, with no segregation, for which the fluidization and sedimentation velocities are equal. Despite a significant discrepancy between the intrinsic properties of the different materials used, all measured velocities are observed to collapse into a single master curve $f\left(\Phi_{s} / \Phi_{\text {pack }}\right)$ provided that they are normalized by the relevant scaling. Regarding to the sedimentation velocity, $\Phi_{\text {pack }}$ turns out to be sufficient to characterize the material made with a random distribution in particle shape and size. Furthermore, these new findings suggest that such fluidized gas-solid mixtures behave as a homogeneous equivalent fluid with a bulk apparent viscosity that only depends on $\Phi_{s} / \Phi_{\text {pack }}$.
\end{abstract}

Fluidization processes are largely developed in both geophysical mass flows and industrial processes in which a swarm of heavy particles is suspended by an upward fluid flow and settles once this flow vanishes. Far from the boundaries, the fluidization and sedimentation processes are considered to be equivalent such as the fluidization velocity $U_{f}$, which maintains the particles at zero average velocity, is expected to be the same than the settling velocity $U_{\text {sed }}$ at which the particles fall when the fluid is at rest. Predicting these velocities represents an important step for the modeling of a wide range of both natural and industrial systems that involve similar suspensions of particles and gas. Many efforts have been previously devoted to the determination of $U_{f}$ in fluidized beds [1-3] as well as $U_{\text {sed }}$ in sedimenting suspensions $[4,5]$. For the simplest case of a population of monodisperse spherical particles, $U_{f}$ and $U_{\text {sed }}$ are found to depend on the fluid properties (its density $\rho_{f}$ and viscosity $\mu_{f}$ ), the particles properties (its density $\rho_{s}$ and diameter $d$ ), the mixture properties (its particle volume fraction $\left.\Phi_{s}\right)$ and the gravitational acceleration $g[1,2]$. Otherwise, when the particles

\footnotetext{
* laurence.girolami@univ-tours.fr

$\dagger$ frisso@imft.fr
} 


\begin{tabular}{c|c|c|c}
\hline \hline Experimental parameters & $A s h^{1}$ & $A s h^{2}$ & $F C C$ \\
\hline \hline Solid particle density $\rho_{s}\left(\mathrm{~kg} . \mathrm{m}^{-3}\right)$ & 1600 & 1490 & 1420 \\
Mean particle equivalent diameter $d(\mu \mathrm{m})$ & 80 & 65 & 71 \\
Particle volume fraction at packing $\phi_{\text {pack }}$ & 0.58 & 0.60 & 0.484 \\
Range of particle volume fraction $\phi_{s}$ & $0.38-0.58$ & $0.40-0.60$ & $0.39-0.49$ \\
Range of dilatation rate $E=\frac{\phi_{p}}{\phi_{s}}$ & $1.06-1.50$ & $1.05-1.50$ & $1.05-1.22$ \\
Minimum fluidization velocity $U_{m f}\left(\mathrm{~cm} \mathrm{~s}^{-1}\right)$ & 0.32 & 0.17 & 0.26 \\
Minimum bubbling velocity $U_{m b}\left(\mathrm{~cm} \mathrm{~s}^{-1}\right)$ & 0.96 & 0.64 & 0.54 \\
\hline \hline
\end{tabular}

TABLE I. Experimental parameters obtained with the different materials $A s h^{1}, A s h^{2}$, and $F C C$.

involved in the mixture are not spherical, their size can be characterized by the diameter of a sphere of the same volume $\vartheta$, such as $d=(6 \vartheta / \pi)^{1 / 3}$, as defined hereafter in this paper. Furthermore, in more common situations, such as those encountered in both natural and industrial systems, the dense suspension usually involves a polydisperse material which makes dependent the mixture behaviour to the particle size and shape distributions. Previous scientific achievements, conducted by one of the authors [6-9], have reported experimental measurements of $U_{f}$ and $U_{\text {sed }}$ for gas-solid mixtures involving both natural and synthetic materials made of volcanic ash (figure 1a, b) and fluid catalytic cracking (figure 1c). These works focused on the dam-break flow of hot dense suspensions and highlighted that the sedimentation velocity plays a major role in the runout duration of basal pyroclastic flows generated by explosive eruptions, such as the gravitational collapse of a lava dome. However, they did not manage to gather the results involving $U_{f}$ and $U_{\text {sed }}$, which were found to depend on the considered sample of particles. The present work revisits these experimental data and shows that a unique scaling allows to match both the fluidization and sedimentation velocities obtained with two different ash samples $\left(A s h^{1}\right.$ and $A s h^{2}$, fig. 1a, b) with those of more spherical synthetic particles $(F C C)$ of comparable sizes distribution but of contrasted geometry (fig. 1c).

Figure 2 illustrates the experimental configuration and the different flow regimes. The particles properties and the experimental operating conditions are listed in Table I, whilst the microscopic overviews of the different samples used, exposed in figure 1, provide the diameter 

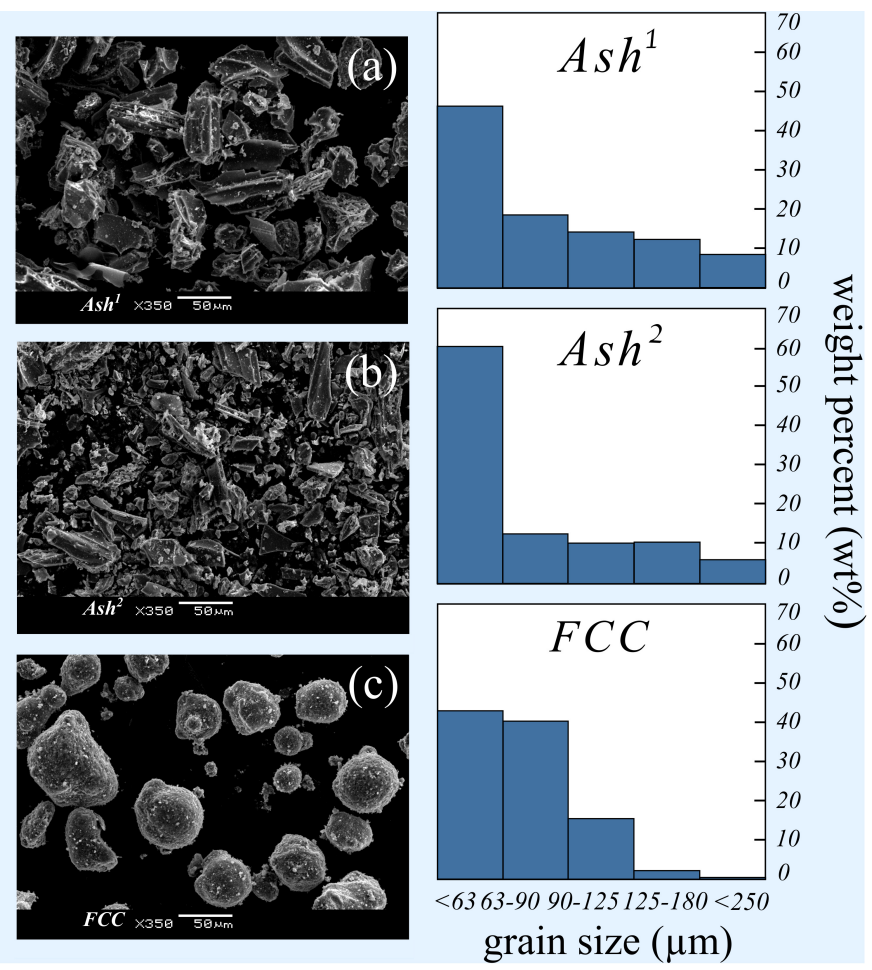

FIG. 1. Representation of the particles size and shape distributions of $A s h^{1}, A s h^{2}$, and $F C C$.
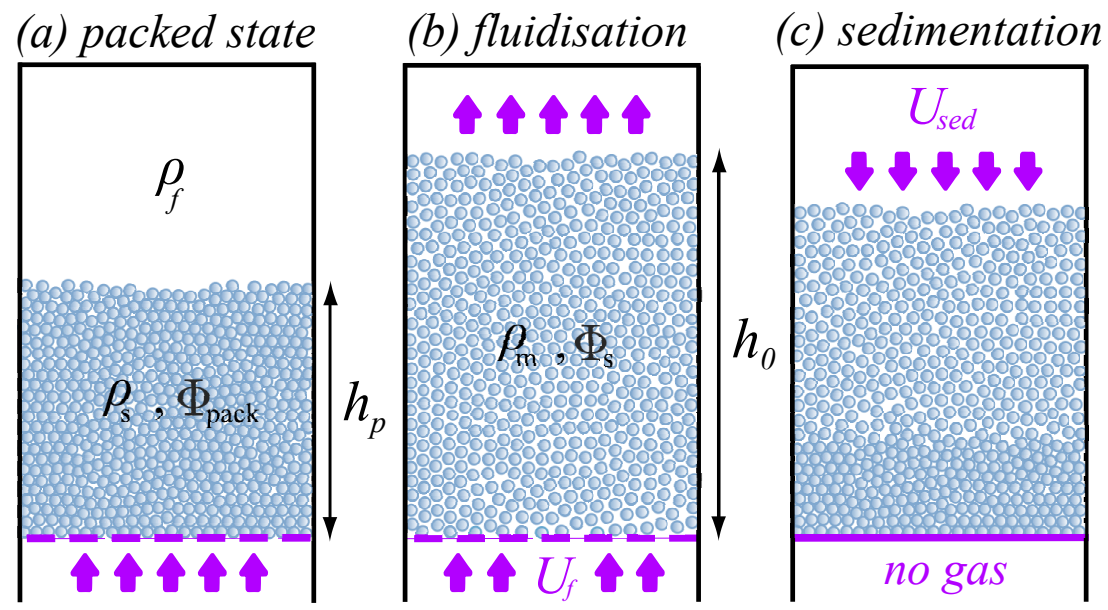

FIG. 2. Fluidization and sedimentation processes applied to a bed of particles: (a) the packed state $\left(U_{f}<U_{m f}\right)$; (b) the homogeneous fluidization associated with a uniform bed expansion $\left(U_{m f}<\right.$ $\left.U_{f}<U_{m b}\right)$; (c) the sedimentation process obtained after cutting the gas supply $\left(U_{\text {sed }}=U_{f}\right)$.

and shape distributions. The reservoir has a rectangular cross-section, $150 \times 300 \mathrm{~mm}^{2}$, much larger than the particle size $(<250 \mu \mathrm{m})$. Experiments have been performed at a temperature of $180^{\circ} \mathrm{C}$ in order to prevent the cohesive effect and agglomeration induced by moisture. 
First, the hot particles were poured into the reservoir and fluidized by a hot gas, injected from a porous plate located at the base. The mixture was homogeneously stirred before cutting the gas injection. The initial state was obtained when all particles have settled down to the reservoir bottom until forming a random loose packing deposit of thickness $h_{p}$ and of solid volume fraction $\Phi_{\text {pack }}$. Fluidization experiments were carried out by injecting gas at superficial velocity $U_{f}$, measured with an accuracy of $\pm 1 \%$ by the means of flow-meters. Increasing $U_{f}$ first amounts to increase proportionally the pressure drop across the static bed whilst $\Phi_{s}$ remains equal to $\Phi_{\text {pack }}$. Above the threshold of minimum fluidization velocity $U_{m f}$, the bed starts to expanse and the pressure drop becomes independent of $U_{f}$ such as maintaining its maximum value. The bed remains homogeneously fluidized provided that $U_{f}$ is inferior to $U_{m b}$, beyond which the nucleation of gas bubbles makes the mixture unstable. The present study reports measurements within the range of values obtained from $U_{m f}$ to $U_{m b}$. Note that the transition between the packed regime $\left(U_{f}<U_{m f}\right)$ and the homogeneous fluidized one $\left(U_{f}>U_{m f}\right)$, when $U_{f} \simeq U_{m f}$, appears quite complex and exposes different behaviours which depend on whether $U_{f}$ is increased or decreased. Sedimentation experiments were carried out by stopping the gas injection once the suspension had reached a given expanded thickness $h_{0}$, corresponding to a given solid volume fraction $\Phi_{s}$. A remarkable feature of this original material, when operated at high temperature, is its expandable properties. Here, the expansion rate, $E=h_{0} / h_{p}=\Phi_{\text {pack }} / \Phi_{s}$, is varied from 1.05 to 1.5 . In this range, no segregation took place during neither the fluidization nor the sedimentation stage, such as the volume fraction $\Phi_{s}$ remains representative to the entire uniform mixture. $U_{\text {sed }}$ was obtained from the time taken for the bed surface to deflate, from $h_{0}$ to $h_{p}$, with an accuracy of $\pm 3 \%$. After sedimentation, the deposit returns to the same initial volume fraction $\Phi_{\text {pack }}$, indicating that this particular loose packing is a characteristic state of each sample which probably keeps memory of the sedimentation process in terms of particles arrangement and orientations.

Grey symbols in figure 3 represent the measured fluidization velocities $U_{f}$ (light grey) as well as the sedimentation ones $U_{\text {sed }}$ (dark grey) as a function of the solid volume fraction $\Phi_{s}$, for the three different samples, while white symbols indicate the measured minimum fluidization velocity $U_{m f}$. These results show that $U_{f}=U_{\text {sed }}$ for all the measurements performed at $U_{f}>U_{m f}$ and indicate that the possible interactions with the vertical walls of the reservoir appear to have a negligible effect on the particles average velocity. This 


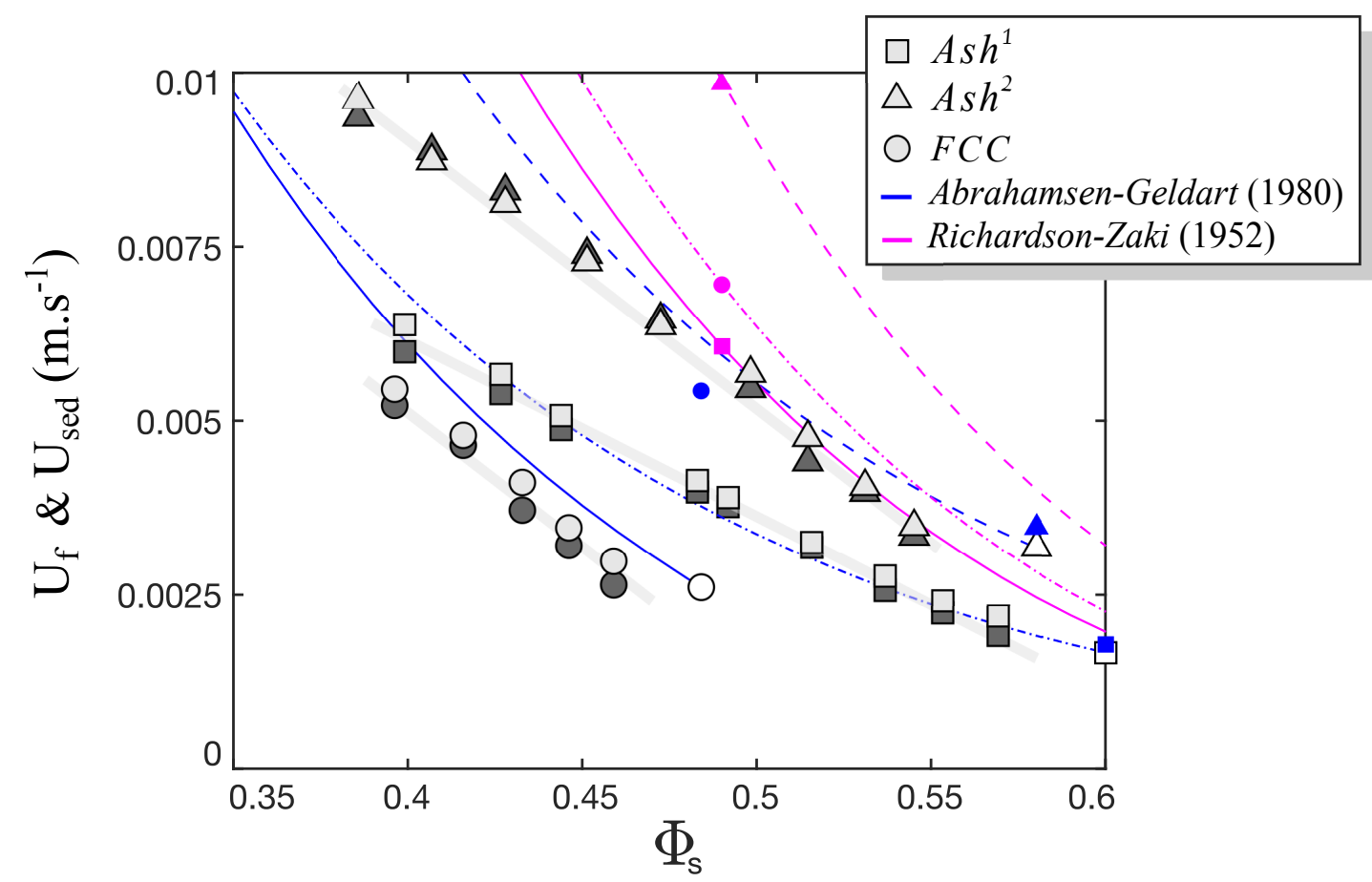

FIG. 3. Fluidization velocity $U_{f}$ (light grey symbols) and sedimentation velocity $U_{\text {sed }}$ (dark grey symbols) as a function of the particle volume fraction $\Phi_{s}$. White symbols represent $U_{m f}$, while blue and pink symbols indicate respectively the predictions of Ergun (1952) [11] and Richardson \& Zaki (1954) [1] for $U_{m f}$.

fondamental finding allows to not distinguish anymore $U_{f}$ from $U_{\text {sed }}$ in the follow of this paper, henceforth referred as $U$. Moreover, the particulate Reynolds number defined as $R e=d U / \mu_{f}$ is lower than 0.03 in all experiments and reflects that the inertial effects are negligible. For each sample, $U$ is observed to decrease, following a constant negative slope, with increasing $\Phi_{s}$ without including the not aligned threshold velocity $U_{m f}$. This observation highlights the presence of a transitional regime that distinguishes the fully fluidized suspensions $\left(\Phi_{s}<0.95 \Phi_{\text {pack }}\right)$ from the partly fluidized ones $\left(\Phi_{s} \simeq \Phi_{\text {pack }}\right)$ in which macroscopic jamming frameworks induced by both inter-particles and particles-walls interactions are developed and play an important role in the mixture dynamics, as observed in granular column collapses initiated from the dense to the loosely packed state [10]. Moreover, the results of the three different samples are observed to be significantly different, even for those obtained with the two samples of volcanic ash, highlighting a lack of universal gathering.

Many approaches have been addressed in the literature to overcome this gap. The mini- 
mum fluidization velocity is commonly described by the Ergun correlation [11],

$$
U_{m f}=\frac{g\left(\rho_{s}-\rho_{f}\right) d^{2}}{150 \mu_{f}}\left[\frac{\left(1-\Phi_{\text {pack }}\right)^{3}}{\Phi_{\text {pack }}}\right],
$$

which was established from a series of measurements performed in packed beds of spherical particles that were sedimented after being fluidized, similarly to the procedure followed in the present study. Predictions of eq. 1 are represented by blue symbols in figure 3. Despite a surprising good agreement observed for the two samples of non spherical particles $\left(A s h^{1}\right.$ and $\left.A s h^{2}\right)$, eq. 1 fails to predict that of the quasi-spherical powders $(F C C)$. An evolution of $U$ extended to the entire fluidized regime above $U_{m f}$ has thus been proposed by [2],

$$
U=U_{m f}+\frac{g\left(\rho_{s}-\rho_{f}\right) d^{2}}{210 \mu_{f}}\left[\frac{\left(1-\Phi_{s}\right)^{3}}{\Phi_{s}}-\frac{\left(1-\Phi_{\text {pack }}\right)^{3}}{\Phi_{\text {pack }}}\right]
$$

Predictions of eq. 2, calculated from our experimental values of $U_{m f}$, are represented by blue (full and dashed) lines. Resulting curves are quantitatively not so far from experimental measurements, since they were initiated from identical values of $U_{m f}$, but fail anyway to reproduce the linear behaviour, especially for $A s h^{1}$. Another famous expression, commonly used in both fluidization and sedimentation literatures, is that introduced by Richardson and Zaki [1] while considering the Stokes regime of dense suspensions,

$$
U=\frac{g\left(\rho_{s}-\rho_{f}\right) d^{2}}{18 \mu_{f}}\left(1-\Phi_{s}\right)^{4.65} .
$$

Predictions of eq. 3, represented by the pink (full and dashed) lines in fig. 3, disagree with the present experiments both in terms of magnitude and evolution trends. Therefore, the velocity scale, $g\left(\rho_{s}-\rho_{f}\right) d^{2} / \mu_{f}$, which is generally considered as the relevant one in the literature, does not allow to collapse the present results. In the aim of attempting to determine the relevant scaling, we consider that the sedimentation of a particle within a concentrated fluidized suspension is equivalent to that described in a homogeneous fluid of density $\rho_{m}=\Phi_{s} \rho_{s}+\left(1-\Phi_{s}\right) \rho_{f}$ and of bulk viscosity $\mu_{m}$. The equation of motion written for the considered particle, falling at velocity $U$ in the equivalent fluid, is given by the balance between the buoyancy and the drag forces :

$$
g\left(\rho_{s}-\rho_{m}\right) \frac{\pi}{6} d^{3}=3 \alpha \pi \mu_{m} d U
$$

where $\alpha$ is a prefactor derived from the Stokes drag force to account for the possible effect of the non sphericity of the particles. Theoretical values of $\alpha$ are known for either oblate 

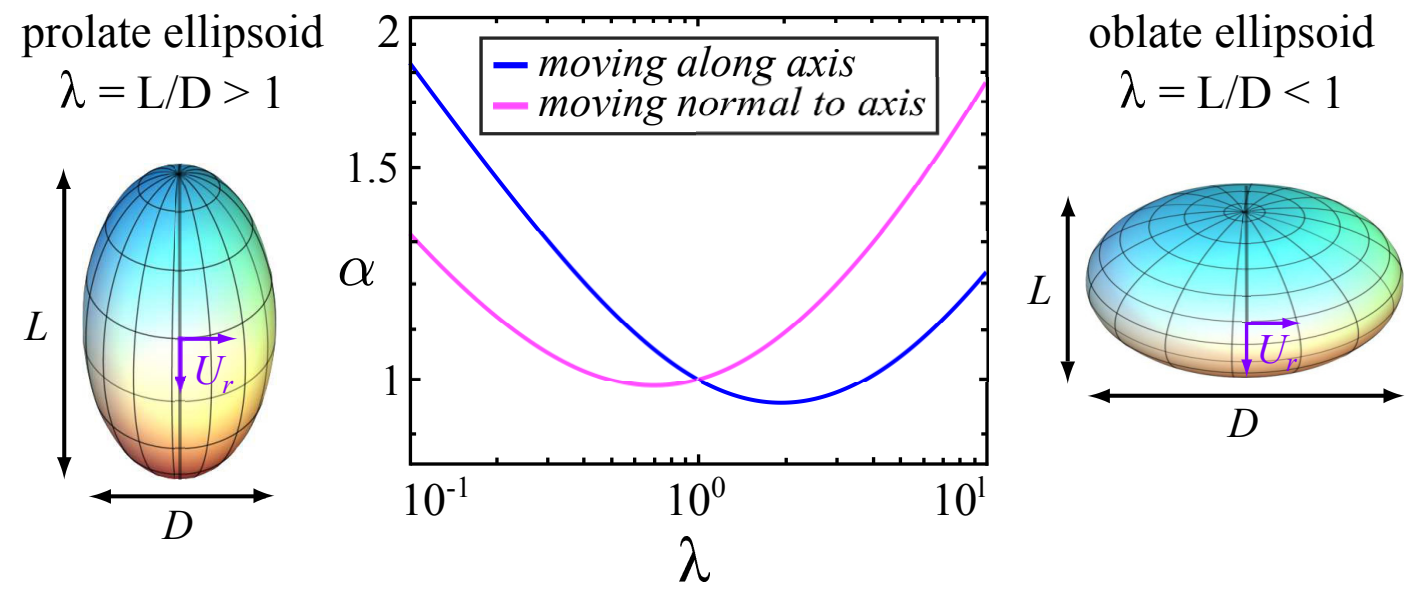

FIG. 4. Correction coefficient $\alpha$ accounting for the non-sphericity of the particles in the Stokes drag force as a function of the particle aspect ratio $\lambda$.

or prolate axisymmetric ellipsoids moving parallelly or normally to their principal axis [12]. Figure 4 shows its value as a function of the particles aspect ratio $\lambda$ and turns out to remain close to unity, even for a rather pronounced gap to sphericity, which leads us to assume reasonably that $\alpha=1$ in what follows.

Thus, equation 4 can be rewritten as,

$$
\begin{aligned}
\frac{\mu_{m}}{\mu_{f}} & =\frac{U_{r e f}}{U} \\
\text { taking } \quad U_{\text {ref }} & =\frac{g\left(\rho_{s}-\rho_{m}\right) d^{2}}{18 \mu_{f}} .
\end{aligned}
$$

Note that the same relation is obtained from eq. 4 by simultaneously considering a density contrast of $\left(\rho_{s}-\rho_{f}\right)$ for the buoyancy, and the relative average velocity between the gas and the particles of $U /\left(1-\Phi_{s}\right)$ for the drag force. Figure 5 highlights that the measured sedimentation velocities, acquired with the three different samples, perfectly collapse into a single master curve when plotted in its dimensionless form $\mu_{m} / \mu_{f}$ as a function of the relevant mixture volume fraction $\Phi_{s} / \Phi_{\text {pack }}$ and drives us to draw two major conclusions. First, the characteristic velocity $U_{r e f}$, built on the density contrast $\left(\rho_{s}-\rho_{m}\right)$, provides the correct scaling for sedimentation (and fluidization) velocities of dense suspensions, when generated in the homogeneous particulate regime of fluidization. Second, the value of $\Phi_{\text {pack }}$ is sufficient to characterize the size and shape distributions of the samples for what regards the determination of $U$.

These results also suggest that the fluidized suspension behaves as an equivalent fluid of 
apparent viscosity $\mu_{m}$. Since the pioneering work of Einstein, many efforts have been devoted to the determination of the bulk viscosity of a dense suspension [see 13, and references therein], which usually propose expressions involving the solid volume fraction $\Phi_{s}$ and its value at packing $\Phi_{\text {pack }}$. For example, [14] derived a theoretical relation for a suspension of spheres which is based on the dissipation of the interstitial fluid between the particles,

$$
\frac{\mu_{m}}{\mu_{f}}=\frac{9}{8}\left[\frac{\left(\frac{\Phi_{s}}{\Phi_{p a c k}}\right)^{1 / 3}}{1-\left(\frac{\Phi_{s}}{\Phi_{p a c k}}\right)^{1 / 3}}\right],
$$

Another popular approach, proposed by [15], consists in extending the dilute regime to the concentrated one in which the viscosity is expected to diverge when $\Phi_{s}$ tends to $\Phi_{\text {pack }}$,

$$
\frac{\mu_{m}}{\mu_{f}}=\left(1-\frac{\Phi_{s}}{\Phi_{\text {pack }}}\right)^{-B \Phi_{p a c k}}
$$

where $B$ is the Einstein's coefficient. Predictions of eq. 7, represented by a pink dashed line in fig. 5, clearly disagree with the present results, whilst Eq. 8 can not either fit the measurements since the dependence of the exponent upon $\Phi_{\text {pack }}$ is in contradiction with the collapse of results involving samples characterized by different $\Phi_{\text {pack }}$. Relaxing the constraint that the viscosity has to recover the value of that of the gas at low $\Phi_{s}$, the following empirical expression, thus only relevant for the dense regime, fits well the experimental data,

$$
\frac{\mu_{m}}{\mu_{f}}=8.6\left(1-\frac{\Phi_{s}}{\Phi_{\text {pack }}}\right)^{-0.45} .
$$

Note however that the physical meaning of such a viscosity is delicate and is expected to differ from that determined in shearing suspensions, which can explain the discrepancy with eqs. 7 and 8. All the more so the rheology of concentrated suspensions is known to be non Newtonian [13], especially for elongated particles [16].

To summarize, we reported experimental data of hot, fine particles fluidized by a hot gas in the Stokes flow regime. The particles have random sizes and complex shapes. Particle volume fractions $\Phi_{s} / \Phi_{\text {pack }}$, ranged from 0.65 to 0.95 , are investigated and correspond to concentrated but still homogeneously fully fluidized suspensions. In this context, no segregation develops and the fluidizing gas velocity is equal to the sedimentation velocity. Despite a wide range of particle size and shape distributions between the different samples, the evolution of the sedimentation velocities against the mixture volume fraction collapse into a single master curve provided that the velocity is scaled by $g\left(\rho_{s}-\rho_{m}\right) d^{2} / \mu_{f}$ and the volume fraction 


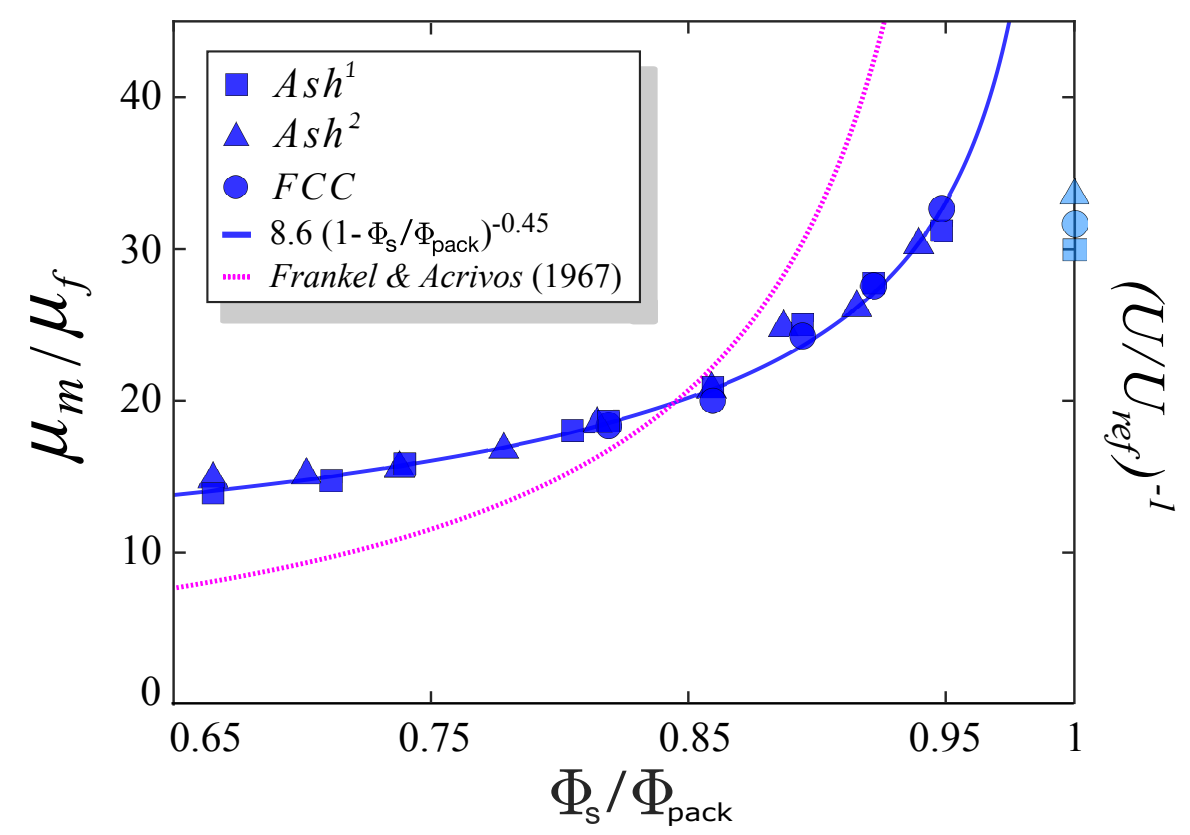

FIG. 5. Apparent bulk viscosity of the suspension normalized by the gas viscosity $\mu_{m} / \mu_{f}$, which is the same than the inverse normalized sedimentation velocity $\left(U / U_{r e f}\right)^{-1}$, as a function of normalized solid volume fraction $\Phi_{s} / \Phi_{\text {pack }}$. Light blue symbols represent the values at $U_{m f}$.

is normalized by its value at packing. This suggests that the gas-particles mixture behaves as an equivalent fluid of density $\rho_{m}=\Phi_{s} \rho_{s}+\left(1-\Phi_{s}\right) \rho_{f}$ and of bulk viscosity $\mu_{m}$, such as $\mu_{m} / \mu_{f}$ only depends on the ratio $\Phi_{s} / \Phi_{\text {pack }}$. The value of $\Phi_{\text {pack }}$, which corresponds to the loose packing state obtained when a previously fluidized suspension has freely settled down to the reservoir bottom, seems therefore to encapsulate all the informations about the size and shape distributions required to reliably determine the sedimentation velocity of different samples. The present results are of primary interest for geophysical mass flows involving highly expanded suspensions of fine hot particles of complex random shape and size, such as pyroclastic flows, as well as for other common chemical engineering systems obtained in comparable environments.

[1] J. F. Richardson and W. N. Zaki, The sedimentation of a suspension of uniform spheres under conditions of viscous flow, Chemical Engineering Science 3, 65 (1954).

[2] A. R. Abrahamsen and D. Geldart, Behaviour of gas-fluidized beds of fine powders part I. Homogeneous expansion, Powder Technology 26, 35 (1980). 
[3] M. A. van der Hoef, M. Van Sint Annaland, N. G. Deen, and J. A. M. Kuipers, Numerical Simulation of Dense Gas-Solid Fluidized Beds: A Multiscale Modeling Strategy, Annu. Rev. Fluid Mech. 40, 47 (2008).

[4] R. H. Davis and A. Acrivos, Sedimentation of Noncolloidal Particles at Low Reynolds Numbers, Annu Rev Fluid Mech 17, 91 (1985).

[5] É. Guazzelli and J. Hinch, Fluctuations and Instability in Sedimentation, Annu. Rev. Fluid Mech. 43, 97 (2011).

[6] L. Girolami, Dynamique et sédimentation des écoulements pyroclastiques reproduits en laboratoire, Ph.D. thesis, Université de Clermont II (2008).

[7] L. Girolami, T. H. Druitt, O. Roche, and Z. Khrabrykh, Propagation and hindered settling of laboratory ash flows, Journal of Geophysical Research 113, B02202 (2008).

[8] L. Girolami, O. Roche, T. H. Druitt, and T. Corpetti, Particle velocity fields and depositional processes in laboratory ash flows, with implications for the sedimentation of dense pyroclastic flows, Bull Volcanol 72, 747 (2010).

[9] L. Girolami, T. H. Druitt, and O. Roche, Towards a quantitative understanding of pyroclastic flows: Effects of expansion on the dynamics of laboratory fluidized granular flows, Journal of Volcanology and Geothermal Research 296, 31 (2015).

[10] L. Rondon, O. Pouliquen, and P. Aussillous, Granular collapse in a fluid: Role of the initial volume fraction, Phys Fluids 23, 073301 (2011).

[11] S. Ergun, Fluid flow through packed colmuns, Chemical Engineering Progress 48, 89 (1952).

[12] J. Happel and H. Brenner, Low Reynolds number hydrodynamics, with special applications to particulate media, Vol. 1 (Martinus Nijhoff Publishers, Dordrecht, 1983).

[13] S. P. Mueller, E. W. Llewellin, and H. M. Mader, Proc. R. Soc. A, Proc. R. Soc. A 466, 1201 (2010).

[14] N. A. Frankel and A. Acrivos, On the viscosity of a concentrated suspension of solid spheres, Chemical Engineering Science 22, 847 (1967).

[15] I. M. Krieger and T. J. Dougherty, A Mechanism for Non-Newtonian Flow in Suspensions of Rigid Spheres, Transactions of the Society of Rheology 3, 137 (1959).

[16] F. Tapia, S. Shaikh, J. E. Butler, O. Pouliquen, and É. Guazzelli, Rheology of concentrated suspensions of non-colloidal rigid fibres, Journal of Fluid Mechanics 827, 725 (2017). 